\title{
Malaria vaccine research and development: the role of the WHO MALVAC committee
}

\author{
Geoffrey AT Targett ${ }^{1 *}$, Vasee S Moorthy ${ }^{2}$ and Graham V Brown ${ }^{3}$
}

\begin{abstract}
The WHO Malaria Vaccine Advisory Committee (MALVAC) provides advice to WHO on strategic priorities, activities and technical issues related to global efforts to develop vaccines against malaria. MALVAC convened a series of meetings to obtain expert, impartial consensus views on the priorities and best practice for vaccine-related research and development strategies. The technical areas covered during these consultations included: guidance on clinical trial design for candidate sporozoite and asexual blood stage vaccines; measures of efficacy of malaria vaccines in Phase IIb and Phase III trials; standardization of immunoassays; the challenges of developing assays and designing trials for interventions against malaria transmission; modelling impact of anti-malarial interventions; whole organism malaria vaccines, and Plasmodium vivax vaccine-related research and evaluation. These informed discussions and opinions are summarized here to provide guidance on harmonization of strategies to help ensure high standards of practice and comparability between centres and the outcome of vaccine trials.
\end{abstract}

Keywords: Malaria, Clinical trials of vaccines, Efficacy measures, Transmission, Modelling, P. vivax vaccine research

\section{Background}

Much has been achieved in controlling malaria in many endemic areas of the world [1,2] and further progress is possible through universal access to and use of existing malaria preventive, diagnostic and treatment measures, including transmission reduction through Anopheles mosquito vector control. Nevertheless, current tools on their own are unlikely to provide elimination in areas of highest transmission, especially in Africa, and new tools are needed. One or more effective vaccines which can be added to available measures could fill that critical gap and could support malaria control. Despite some investment in research on the development of malaria vaccines over the past thirty years [3,4], currently only one candidate vaccine, RTS,S/AS01, has reached the stage of phase III clinical trials, with the prospect of being submitted for consideration of licensure soon [5]; several others have been tested in phase II field trials [6].

Throughout this period of increased vaccine effort, WHO has had an important role in supporting various aspects of basic research projects through the $\mathrm{WHO} /$

\footnotetext{
* Correspondence: geoff.targett@lshtm.ac.uk

${ }^{1}$ Faculty of Infectious and Tropical Diseases, London School of Hygiene \&

Tropical Medicine, Keppel Street, London WC1E 7HT UK

Full list of author information is available at the end of the article
}

UNDP/World Bank Special Programme (WHO/TDR) and has also played a major normative role in providing guidance on many aspects of vaccine development. Landmark meetings were held to highlight the need for improved adjuvants, by bringing together representatives of groups involved in developing and testing new potential products, recognizing that malaria antigens were often involved in the first wave of testing novel agents [7]. WHO worked with funding agencies to convene meetings of scientists, regulators and others to review the state of the art of vaccine development, reviews of key ethical issues [8], and maintains an ongoing record of malaria vaccines under development (known as the Rainbow Table) [3]. Perhaps most importantly, WHO has worked with its advisory committees to gather best evidence then provide guidance for vaccine trial designs that ensure that results generated are relevant for establishing subsequent policy recommendations, to assist national programmes in malariaendemic countries.

WHO's advisory committees are a key mechanism for identification of research priorities for immunization and for development of consensus-based guidance on clinical development and testing of vaccines. The WHO Malaria Vaccine Advisory Committee (MALVAC) succeeded

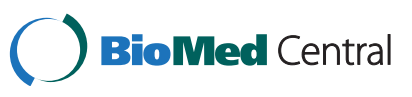

(c) 2013 Targett et al.; licensee BioMed Central Ltd. This is an open access article distributed under the terms of the Creative Commons Attribution License (http://creativecommons.org/licenses/by/2.0), which permits unrestricted use, distribution, and reproduction in any medium, provided the original work is properly cited. 
IMMAL and VDR Committees, and provides advice to WHO on strategic priorities, activities and technical issues related to global efforts to develop vaccines against malaria, with emphasis on the public health needs of developing countries. In the five years 20082013 MALVAC has convened eight key meetings of experts, together with several working groups, whose detailed assessments have provided valuable consensus views on priorities and best practice for selected vaccine-related research and development strategies. By providing the leading independent global malaria vaccine forum for funding agencies, sponsors and investigators, WHO has increased collaboration between key malaria vaccine R\&D stakeholders. WHO has also improved the comparability of key endpoints by convening technical groups to provide consensus based protocols and Standard Operating Procedures, ensuring extensive consultation amongst stakeholder groups. The technical areas for these consultations have included design and conduct of sporozoite challenge trials, Standard Operating Procedures for malaria microscopy in challenge trials, [9] optimization of clinical challenge trials for asexual blood stage vaccines, measures of efficacy of malaria vaccines in phase IIb and phase III trials, workshops on standardization of malaria vaccine immunoassays, evaluation of assays and trial designs to be applied to interventions against malaria transmission, development of whole organism vaccines for malaria endemic countries, and priorities in research and development of vaccines for Plasmodium vivax and their evaluation. Recently, WHO highlighted the need for informationsharing among HIV, TB and malaria vaccine communities and working with NIAID convened a technical forum on heterologous prime-boost immunization across the three diseases [10]. Furthermore the MALVAC committee will have a key role in advising WHO on the updated version of the Malaria Vaccine Technology Roadmap [11].

These informed and impartial opinions and recommendations are summarized here to provide guidance on harmonization of strategies that will help to ensure high standards of practice, and comparability between centres and the outcome of vaccine trials. Reference is made to reports of the meetings (and to selected other relevant publications) that provide the more detailed discussions on which the recommendations are based. The text below summarizes the reports of individual meetings and should not be considered to be the position or policy of the WHO. For outcomes of individual meetings, readers are directed to the individual meeting reports.

There is a distinct WHO advisory mechanism which provides advice on vaccine candidates in advanced development and approaching possible availability for use. This is known as the Joint Technical Expert Group (JTEG) on malaria vaccines, reporting jointly to the WHO Strategic Advisory Group of Experts on Immunization, and to WHO's Malaria Policy Advisory Committee [12]. MALVAC will continue to provide advice to WHO on the longer term malaria vaccine $R \& D$ considerations, with JTEG available to provide evidence reviews for possible policy recommendations when products become sufficiently advanced.

\section{Consensus-based guidance on clinical trial design}

A vital part of the development of candidate malaria vaccines is the careful planning of all phases of clinical trials, always with a view to enhancing comparability between vaccine clinical trials, sites, and alternative development programmes. A second important objective is generation of data to terminate or advance projects appropriately. Phase I trials are used to determine whether candidate vaccines have the required profile of safety and immunogenicity, with Phase IIa trials in malaria designed to provide actionable information on efficacy, safety and immunogenicity including controlled human malaria infection. They are screening trials intended to select candidate vaccines to take forward into field trials, and to select which vaccine formulations to terminate. These proof of concept studies have usually started with adults in non-endemic areas before moving to the target group of children of an endemic area. Phase IIb and III proof of principle field trials require progressively larger numbers of subjects depending on the primary endpoint to be measured and the controls necessary for comparison. Phase III trials are traditionally designed with the primary objective of providing data suitable for regulatory filing. However a focus in malaria vaccines has been to ensure that data are also suitable for evidence-based public health policy recommendations as far as possible. This can avoid the need for additional Phase III trials.

\section{Challenge trials in malaria-naïve volunteers Sporozoite challenge trials for pre-erythrocytic and blood-stage vaccines}

Controlled Human Malaria Infections (CHMI) [9,13] are used to assess candidate vaccine efficacy in malaria-naive individuals. Challenge trials in volunteers are important as they inform future clinical trials - whether or not to proceed; dosing, route, schedule and vaccine presentation. This allows iterative improvement of the vaccine construct and its use. Field trials for pre-erythrocytic vaccines should be dependent on first achieving a pre-determined level of efficacy in challenge trials. Future efficacy field trials of new vaccine candidates or combinations may well have to be non-inferiority trials (if RTS,S, for example, is licensed) in regions of reduced transmission, potentially requiring very large sample sizes, thus making them costly and complex with subjects enrolled in several centres. The need to achieve greater confidence in expectations of efficacy prior 
to initiating multi-million dollar field studies in large numbers of individuals means that even greater reliance may be placed on carefully standardized CHMI studies. They will also be important for the same reasons in go/no go criteria and prioritization of blood stage vaccines (Figure 1). The significant additional challenges with $P$. vivax challenge trials are considered later.

\section{Blood stage challenge trials}

As an alternative to sporozoites, parasitized erythrocytes can be used to challenge vaccinated individuals [14]. They may have advantages over sporozoite challenge for testing the efficacy of blood-stage vaccines since they allow use of a standardized and low dose challenge and, consequently, a longer period for assessment of induced

\section{Screening of potential participants}

- A pre-requisite is very careful selection of potential volunteers involving extensive health screening, including mental health and specific infections, disorders, or concurrent treatments that could compromise the outcome of the trials.

- The safety of the volunteers must be paramount throughout the study and they must be fully informed about the nature of the trial and kept up to date with its progress as it affects them.

Parasite and mosquito strains

- The recommended procedure includes use of a Plasmodium falciparum malaria isolate, or one of its clones, that is adapted to culture. All must have defined sensitivities to effective drug treatments, and blood donors for parasite culture need screening for known infectious agents. Banks of parasites are available.

Laboratory bred anopheline mosquitoes have been used. Safety procedures are again the highest priority.

Infectivity controls and challenge

- A control (non-vaccinated) group of volunteers should be included. All volunteers are exposed to a fixed number of infected mosquitoes (five) for a defined period of time within totally secure insectaries. For efficacy analysis, trial size is greatly impaired if even one of the control subjects does not develop patent parasitaemia, hence a large dose is given which may represent an unnaturally high (artificial) challenge.

- Recently, mass-produced purified, GMP standard cryopreserved sporozoites have been assessed for human challenge trials[13].

Follow-up

- A precise schedule of follow-up is required to cover the period of possible acute allergic reactions or other adverse events, and the onset of malaria infections that require prompt optimal treatment.

- Formal outreach plans are required in the event that a volunteer does not attend for follow-up prior to receiving curative treatment.

\section{Endpoints}

- The primary endpoint is first detection of patent parasitaemia, usually as determined by microscopy, measuring parasites per unit volume in thick blood films. Fully developed and agreed standard operating procedures (SOP) that take account of local variations in procedure but give a standardized outcome measure have been developed[9]. The expertise required by clinical trial microscopists is distinct from that needed for diagnosis in malaria-endemic regions, since the requirement is to be able to detect the earliest stage of patency, whilst avoiding false positives.

- A secondary endpoint is the pre-patent period, the time interval between challenge and the first occurrence of patent parasitaemia, which can vary depending on both parasite and host characteristics.

- Increased frequency of blood sampling (e.g. twice daily), in order to measure accurately peripheral blood parasite density and pick up even modest efficacy, particularly in blood stage vaccine trials, should be employed.

- Molecular detection methods (PCR) are an important supplement to blood film diagnosis, and may replace microscopy as the standard in some centres in the future. These molecular genetic techniques allow more precise calculation of growth rate, and have decreased the detection threshold for asexual parasitaemia substantially. Harmonization of PCR methods is needed to ensure comparability, and the establishment of a reference centre and repository may be beneficial.

- Evaluation of parasite growth curves can be helpful for assessment of efficacy that relates to reduction in numbers of parasites leaving the liver.

- Immune correlates of protection are not yet fully defined. CHMI allows these to be explored and opportunities should be taken to do so.

Figure 1 Standardization and conduct of $P$. falciparum sporozoite controlled human malaria infection trials. CHMI is used to denote sporozoite challenge but it is also sometimes used too for blood-stage challenge. 
blood stage immunity before parasites are detected and treatment is required. In contrast, the large sporozoite challenge required to ensure that every control subject is infected may lead to heavy infection and a large numbers of parasites leaving the liver and, as a consequence, earlier detection of parasites requiring treatment.

Rigorous safety depends on having a well-characterized source of erythrocytes free from adventitious agents that meet stringent blood product safety requirements. This is the area of particular concern that for some investigators may still outweigh the perceived benefits of blood-stage challenge. The antigenicity of infected red blood cells in vaccinated volunteers is also a safety issue to be considered. Quantitative PCR monitoring of infection is valuable as it provides a detection threshold of asexual parasitaemia of approximately 20 parasites $/ \mathrm{ml}$, well below that possible by microscopy, and potentially increases the time for observations and assessment of efficacy before treatment is required.

There are, however, some drawbacks to use of bloodstage challenge. The challenge bypasses skin and liver stages of infection and removes the possibility of detecting protective cellular immune responses against the late liver stage or antibody against merozoites released from the liver. A low inoculation of parasitized erythrocytes has no counterpart in naturally acquired blood stage infection. Further development of blood stage challenge trials and comparisons with sporozoite challenge are desirable. Development of multiple antigenically distinct parasite strains for evaluation of heterologous protection is also required. Independent evaluation of methods used for modelling parasite growth curves should be made to assist decision-making.

\section{Phase IIb and Phase III malaria vaccine trials}

Detailed recommendations were made on the implications of different measures of efficacy as they affect vaccine impact, comparability of trials, licensure and wider public health benefits [15]. The conclusions were:

- Field evaluation of a vaccine has to be done in the context of other control measures. Assessing efficacy is complicated for malaria where first infection (or vaccination) gives only partial protection against re-infection and the same individual may have multiple episodes of clinical malaria. The primary measure of efficacy is commonly reported as the incidence of first episode of infection or of clinical malaria. This takes no account of subsequent episodes and, from a public health perspective, reduction in the total number of events in some defined time period following vaccination is more relevant than measuring time to first event as the primary efficacy endpoint.
- Incident malaria infection is a prerequisite of clinical malaria but, additionally, there can be incident infections without clinical symptoms. If protection against incident malaria infection could be used as a correlate for protection against clinical malaria, this would allow smaller trial sizes and less cost (in malarious areas where many trials are likely to be conducted, incidence of infection is high, with the vast majority of susceptible individuals expected to experience infection in a one to three month period at the peak of local transmission).

- A high incidence of malaria and heterogeneity of exposure make estimates of efficacy difficult. In particular, waning of efficacy and heterogeneity in exposure cannot be distinguished by measuring the proportion of individuals remaining disease free at different times after vaccination. Boosting from natural infection should be evaluated. Trial designs in endemic countries will need to be able to detect duration of protection at least up to two years after vaccination, and to rule out deferred or rebound increases in mortality as a consequence of increasing susceptibility following a period of vaccine-induced immunity.

- Evaluation of efficacy in the context of existing malaria preventative interventions is a priority. The dosing schedules required for successful vaccination may have implications for feasibility of scale-up. Possible interference with EPI vaccines in infants should be assessed. The possibility of reduced transmission in some field trial sites will need to be addressed in field trial design; larger enrolments may be required, and because age at risk will have extended beyond the ages for EPI, vaccines may also need to be administered to the older children at risk.

- Safety, immunogenicity and efficacy should be established prior to a vaccine being given to specific high risk groups (e.g. infants, in pregnancy, and immune-compromised individuals), Monitoring and evaluation plans should include both pharmacovigilance and a sustainable disease burden monitoring system. However these pose major challenges in many malaria-endemic settings.

- Impact on malarial transmission (see below) is a key part of assessment of any potential new malaria intervention, although it may not be included in the first Phase III trial. The future combination of single or multi-component candidate malaria vaccines with other malarial interventions should be considered.

- As a general rule methods for design and analysis of Phase III trials should be registered in publicly available data bases before results are unblinded. Data sharing will increase understanding of the 
likely public health impact of a new vaccine in the context of existing control measures.

- Post-licensure, data should be acquired on long-term effectiveness in multiple transmission settings and changing control measures and epidemiology, noting the consequences when immune individuals have long periods of reduced exposure, for example whether they become more susceptible to the consequences of later episodes of malaria. The long-term safety must also be evaluated.

\section{Measures of efficacy of interventions against malaria transmission}

Reduction in transmission remains the fundamental goal of malaria control and measuring changes in transmission allows a better understanding of the interactions between different interventions (vector control, treatment, vaccination) and their combined impact. Some currently available anti-malarial interventions lead to a reduction in transmission even in highly malarious areas, and transmission reduction is the key metric in a malaria elimination strategy.

Better measures of transmission are needed but, as yet, there are no agreed and standardized measures of malaria transmission and these may need to be different in areas of high and low transmission. Measurement of transmission is costly and time-consuming, requiring repeated observations. In some very low transmission areas, incident infection leads to disease and may, therefore, be a surrogate for ongoing transmission. However, in other areas of very low transmission, substantial numbers of asymptomatic infections have been detected with sensitive molecular tools. At low transmission intensity (for example in some of the environments in which transmission blocking vaccines are likely to be tested and introduced) estimates have very large confidence intervals and surrogates are required (see below). Transmission blocking vaccines (TBV) specifically target sexual or sporogonic stage parasites, or mosquito midgut antigens. However, the so-called vaccines that interrupt malaria transmission (VIMT) may have their primary effect on other of the three main life-cycle stages but, additionally, may have a significant indirect effect on transmission.

The key effect required for sexual stage or mosquito antigen targets is substantial reduction in the proportion of infected mosquitoes. By contrast, the key effect required for pre-erythrocytic vaccines when viewed as VIMT, is a major reduction in the proportion of humans carrying sexual stages.

A WHO MALVAC meeting considered measures of efficacy of anti-malarial interventions against malaria transmission [16]. The objectives were to evaluate current methods for measurement of malaria transmission and assessment of assays and clinical trial designs that should be applied to measurement of reduction of transmission (of Plasmodium falciparum). The measures considered necessary included:

(i) Epidemiological (incidence of new infections)

(ii) Standardized assays of transmission from humans to mosquitoes (Figure 2) [16,17]

(iii) Entomological (estimating new human infections by mosquito measures)

(iv) Surrogate serological and molecular measures, e.g. sero-negativity in young children as transmission declines.

A major gap in knowledge is the lack of understanding of the relationship between a result in a trial in a group of vaccinated individuals (each one having a different percentage reduction in oocysts in a different percentage of mosquitoes fed on that person), and the potential effect on transmission. It may be that the most important figure is the proportion of vaccinated individuals who are rendered unable to be a source for feeding mosquitoes to become infectious; another parameter may be the duration of infectiousness of vaccinees.

\section{Heterogeneity in malaria transmission and focal transmission (hotspots)}

There is spatial and temporal heterogeneity in malaria transmission in both low and high transmission endemic areas, seen particularly in incidence of infection in infants and in population based sero-positivity rates. High-density carriers of gametocytes transmit more often in feeding experiments but, importantly, sub-microscopic gametocyte carriers may account for a substantial proportion of transmission. Molecular techniques are required for detection of sub-patent infections as microscopy and rapid diagnostic tests (RDT) miss many asexual blood stage parasites and gametocytes. Targeting young children alone with transmission-reducing interventions is unlikely to be successful, as older children and adults also infect mosquitoes.

\section{Entomological measures of transmission}

The two key measures used to evaluate the impact of interventions are the entomological inoculation rate (EIR), the number of infectious bites per person per unit of time (day, year), and the daily rate of potential transmission by a mosquito population known as the vectorial capacity. Human landing catches (HLC) are used as the standard for mosquito collection but subjects vary in their attractiveness and there are ethical concerns. Light traps are used as an alternative and to compare indoor/ outdoor biting and time of biting. They lack reliability outdoors and the mosquito composition may vary from HLC. 
1. Direct feeding assay (DFA)

- Laboratory reared mosquitoes are fed on naturally exposed individuals.

- Some ethical committees have expressed concerns about DFA especially when children are involved, others continue to approve use of DFA in adults.

2 Direct membrane-feeding assay (DMFA)

- Blood samples drawn from naturally infected humans are used for membrane feeding of mosquitoes.

- An advantage is it provides assessment of field derived parasite isolates.

- Control results (a measure of inherent infectivity) are determined by replacing autologous plasma with nonimmune serum prior to feeding.

- The assay is difficult to standardize

\section{Standard membrane feeding assay (SMFA)}

- Uses sera or purified immunoglobulins, in vitro cultured gametocytes, and laboratory strains of Anopheles mosquitoes.

- It is valuable for both pre-clinical and clinical studies.

- Threshold criteria (Go/No-Go) can be set.

- Shortcomings include: limited sensitivity; variations in oocyst numbers between experiments; limited number of suitable cultured parasite strains. There is uncertainty about the relative importance of: percentage of individuals rendered non-infectious; percentage of mosquitoes rendered non-infectious after feeding; reduction of parasite numbers, and the relation between any of these measures and effects on transmission at population level.

- Greater standardization is under investigation. The suggested output is a control/test ratio for each test to minimize effects of variability.

\section{Non-clinical assays using transgenic rodent malaria parasites that express $P$. falciparum sexual} stage genes[17].

Figure 2 Assays for evaluation of reduction in transmission from humans to mosquitoes. A priority is for assays with acceptable precision to improve comparability between trials and to assist regulatory authorities.

The minimum EIR measureable reliably is $5-10$ and transmission-blocking vaccine trials are likely to be conducted at field sites with low to moderate EIR. Nonrandom distribution of vectors, as well as endophilic or exophilic behaviour, present problems. Serological measures of exposure of humans to Anopheles salivary peptides can be used to measure changes such as the impact of ITNs on biting. ELISA measures of sporozoite infections in mosquitoes are convenient but some reports have indicated a high level of false positivity which gives an overestimate of infected mosquito rates. Measuring the infection rate according to age in wild caught mosquitoes requires labour intensive age grading and large numbers of mosquitoes.

\section{Clinical trials of transmission-blocking vaccines Phase $l a, l b$ and $I l b$ vaccine studies}

Safety and immunogenicity and functional activity will be required, as already indicated, before progression from Phase I in naïve volunteers to endemic country trials. Intermediate proof of principle may be shown by demonstrating antibody-mediated reduction in infectivity of humans to mosquitoes in membrane-feeding assays. Efforts to validate or quantify the assays are required and, currently, Go/No-go criteria from Phase I to Phase IIb trials are not agreed. The possibility of demonstrating a reduction in infectivity of vaccinated humans to mosquitoes in Phase IIb also needs further standardization of study design and analysis.

\section{Phase III design for a malaria TBV}

Field trial sites for phase III trials need to be characterized in terms of parasite prevalence and heterogeneity (hotspots), infectious reservoirs, incidence of infection or clinical malaria in infants; mosquito exposure; serology and natural transmission-blocking immunity. There are challenges for design of clinical trials in both high and low transmission sites as an effect may be difficult to show in one case, and power may be lacking to show an effect in the other. Furthermore, transmission may change over the several years of the study. Trial design will be complex. Cluster-randomized efficacy trials may be required, and a step-wedge design has also been suggested.

It is important to distinguish between those interventions with expected direct effects on other stages of the life cycle and possible indirect effects on transmission (VIMT), and interventions only expected to have effects 
on transmission (TBV). Cluster randomized trials can be used for any VIMT, measuring both the direct and indirect effects, as well as for TBV. Contamination amongst clusters is a problem and a $2-4 \mathrm{~km}$ buffer zone was recommended during a MALVAC consultation.

Surrogate endpoints for assessing transmission-blocking vaccines in humans were proposed [16] using $e$ g. oocyst prevalence in membrane feeding. Whether conducted preor post-licensure (i.e. Phase III or Phase IV), trials designed to evaluate the effect of a TBV on transmission will be necessary and could incorporate the surrogates or biomarkers as additional endpoints. The primary endpoint for a Phase III trial of a TBV would be incidence of human infection, and sensitive assays for detection of infection are necessary for such a purpose. Secondary endpoints may include incidence of clinical cases and entomological measures such as EIR, although it should be noted that in trial settings, transmission might be close to the lowest transmission level at which EIR measurements are reliable. Tertiary endpoints derived from serological or nucleic assay measures become important for surveillance-related operational research.

Age groups should always include children through to adolescents, but further research and modelling on the distribution of the infectious reservoir will be required to determine whether this needs to be extended to young adults or even the whole population (except groups who could be at high risk of vaccine side-effects). A follow-up of at least two years is required.

\section{Transmission-blocking vaccine development}

Good progress has been made using different approaches to development of transmission-blocking vaccines that target sexual or sporogonic stages of the life cycle. Candidate vaccines expressing antigens of $P$. falciparum involved in fertilization induced strong transmission-blocking activity assessed with standard membrane feeding assays [18]. Various vaccine formulations of a post-fertilization molecule had equally high transmission-blocking activity [19,20]. Conjugates have been developed for phase I trials and formulation of vaccines to further enhance their immunogenicity is under investigation. Combination vaccines with pre-erythrocytic and sexual stage components are planned. Preferred product characteristics require further work.

\section{Modelling impact of anti-malarial interventions}

Leading modelling groups combined to present opinions on the questions relating to impact of interventions that are best answered by modelling, the outcomes of particular interest to show impact on transmission, and what field data are necessary to make models more robust [16]. It was strongly recommended that field researchers work with modelling groups to improve outputs as the models need more information on:
- different levels and heterogeneity of transmission;

- the influence of superinfection on duration of infection and infectivity;

- the infectious reservoir and age structure in low transmission settings;

- the rate of acquisition of immunity in different transmission settings and loss of immunity when transmission is reduced;

- vector species and densities;

- the relationship between transmission and climate;

- human behaviour and socio-economic factors.

There is a large discrepancy between estimates of $R_{o}$ in high transmission settings measured by the number of infections per person per unit of time-the force of infection (FOI), or by the entomological inoculation rate (EIR). The effect of a TBV can be measured by the proportionate reduction in probability that a mosquito acquires infection at a given feed. Prevalence of infection in mosquitoes is probably more important than density of infection.

Specific TBV may not be better at interrupting transmission than other vaccines, all of which could have the additional benefit of an indirect effect on transmission. However, chances of elimination depend on coverage, initial transmission level, good surveillance, natural immunity and human population size. Vaccines need consideration in terms of both transient dynamics and effects over time. Transmission is heterogeneous with respect to intensity, seasonality, vector species and foci of infections. Modelling should be able to accommodate this heterogeneity together with human infectiousness and immunity in order to inform discussions about who and when to vaccinate with a transmission-blocking vaccine and which other interventions should be included [21]. Sensitivity analyses are important to provide information on key parameters driving uncertainty in the models. Further transmission-related epidemiological studies may be very helpful for data fitting, depending on the study design.

\section{Whole organism malaria vaccines \\ Attenuated sporozoite vaccines}

Humans exposed to bites of large numbers of infected irradiated mosquitoes have shown a high level of shortterm protection in controlled human malaria infection trials [22-25]. This has provided a basis for research into the development of attenuated sporozoite vaccines. An irradiated sporozoite production programme using GMP procedures has been developed and has made available clinical grade materials for phase I/IIa vaccine trials [26]. Proof of concept studies have begun in adults with radiation-attenuated vaccines [27].

Considerations that require evaluation and further research include the size of the vaccine dose, the 
number of doses and their timing, routes of administration, longevity of protection, boosting, immune correlates of protection, protection against heterologous challenge, and storage, stability and transport of the vaccines. Genetically-attenuated sporozoites potentially provide an alternative to irradiated sporozoites as vaccines but the possibility of reversion to virulence or under-attenuation with both irradiated and genetically attenuated sporozoites requires careful assessment [28].

\section{Sporozoite inoculation and chemoprophylaxis}

Volunteers exposed to bites of mosquitoes infected with $P$. falciparum while simultaneously given chloroquine prophylaxis were fully protected against a challenge infection given 2.5 years later opening a new approach to studies on mechanisms of pre-erythrocytic protective immunity [29].

\section{Asexual blood stage whole organism vaccines}

Low dose parasitized erythrocyte inoculation, leading to infection controlled by chemotherapy, can provide protection against challenge infections in malaria naive volunteers without induction of detectable antibody [22,30]. A key safety requirement is a constant source of erythrocytes and other blood products free from adventitious agents. The antigenicity of infected red blood cells in vaccinated volunteers needs to be ascertained but has not been a problem so far. This system of "experimental medicine" can provide insights into protective immune responses induced, and antigenic correlates of protection to guide later production of defined antigen vaccines, even if the concept does not progress as a candidate vaccine in its own right. Issues identified for further studies include whether antibody in malaria-exposed individuals would interfere with the vaccine and whether adjuvants are required to help promote the desired immune response.

\section{Plasmodium vivax vaccine-related research}

Much of the vaccine-related research and planning is focussed on P. falciparum malaria yet the burden of disease due to $P$. vivax is high. The global population at risk of $P$. vivax has been estimated at over 2 billion [31]. Outside Africa, $P$. vivax accounts for more than 50 per cent of malaria cases. There are wide uncertainty ranges around global disease burden estimates for $P$. vivax, although it is agreed that $P$. vivax represents a major cause of disease in Asia and South America in particular. There is increasing evidence for $P$. vivax infection in more settings in Africa [32].

Two WHO MALVAC meetings considered priorities in research and development of vaccines against $P$. vivax [33] and provided detailed guidance on the evaluation of $P$. vivax vaccines in naturally exposed populations [34].

\section{Research required for development of vaccines against vivax malaria}

Plasmodium vivax has aspects of its biology and life cycle that are species-specific when compared with $P$. falciparum and these make all control measures more difficult: infectious gametocytes appear early in the infection; development in the liver includes formation of persisting hypnozoites; the extrinsic cycle is shorter and is completed at a lower temperature; there is efficient transmission even when mosquitoes are highly seasonal. Plasmodium vivax and $P$. falciparum are commonly sympatric, particularly where transmission of $P$. vivax is high. There is conflicting evidence on the impact of one species on the other.

Detailed baseline studies that are required include: burden of disease; the clinical spectrum and incidence of severe malaria and mortality; relapse patterns; strain heterogeneity; interactions with other species and seasonality of $P$. vivax and $P$. falciparum; immunology specific to $P$. vivax and in mixed infections; economic analyses. These will guide determination of sample size in trials, timing of vaccination, and surveys in relation to transmission.

Data are required particularly on sensitivity to drugs needed for radical treatment, especially to primaquine, and on the rate of G6PD deficiency. No radical cure treatment is fully effective. Though pre-erythrocytic and asexual blood-stage vaccines are needed, special consideration of the role of vaccines in blocking transmission is required. In the long-term quest for eradication, the MalERA consultation [35] acknowledged that further basic research to achieve this goal must include as priorities continuous in vitro culture of asexual blood stage forms and gametocytes, and hypnozoite production in relevant cell lines. Non-human models of $P$. vivax are available, but these match human infections in variable ways and should, therefore, be chosen according to the questions being asked.

\section{Clinical trials}

Plasmodium vivax presents some substantial additional challenges when compared with P. falciparum; these are highlighted below. It is noted that testing for protection against hypnozoites is currently not possible.

\section{Controlled human malaria infection trials}

Like P. falciparum, a standardized human sporozoite challenge model is required for evaluation of pre-erythrocytic vaccine candidates. However, it is not yet possible to challenge with the same highly characterized clone of $P$. vivax due to the lack of continuous asexual and gametocyte in vitro culture systems. Challenges have been conducted with varying numbers (3-9) of mosquitoes infected with different $P$. vivax strains. Challenge studies thus include the additional issues of challenge with wild type parasites and the possibility of relapses. There are plans to produce vialled, cryopreserved $P$. vivax sporozoites for a needle- 
based $P$. vivax challenge model (cf $P$. falciparum). As with $P$. falciparum, Phase IIa trials are helpful for preerythrocytic vaccines, but somewhat contentious for bloodstage vaccine candidates.

\section{Phase IIb and Phase III trial designs for Plasmodium vivax}

The sequence of trials required is similar to that for $P$. falciparum. With $P$. vivax, vaccine profiles are based on the need to identify the target groups:

(i) to prevent infection in all age groups in low endemic areas. Mass vaccination may be aimed at elimination or outbreak control.

(ii) to prevent disease in infants and children in high transmission areas - where P. vivax and P. falciparum infections are commonly co-endemic.

(iii) to vaccinate migrants, who need long-term protection to cover relapses.

Initial Phase IIb/III trials should be in highly endemic areas (in children under five years of age). Later trials need to include additional target groups, according to the endemicity of infection. Major challenges to study design and sample size calculations include individuals who have asymptomatic parasitaemia, hypnozoite infections, and P. falciparum co-infections that require treatment. Virtually nothing is known about immunity to hypnozoites and assessment of their presence is currently not possible; meaning that production of a highly desirable therapeutic vaccine is so far out of reach.

For pre-erythrocytic vaccines, a treatment-reinfection design (involving radical treatment to remove hypnozoites) is best if the primary endpoint is incidence of infections. It is difficult to detect mixed infections without molecular techniques. Radical cure to eliminate hypnozoites does however present problems (e.g. haemolytic effects, compliance, and not being fully effective). For blood stage vaccines radical cure is not required if the vaccine will protect equally well against relapse infections.

\section{Plasmodium vivax efficacy endpoints and follow-up}

It is proposed that, for first pre-erythrocytic vaccine trials, incidence of infection is the primary endpoint required. For subsequent trials, incidence of disease should be determined. For blood stage vaccines, the primary efficacy endpoint needed is the incidence of uncomplicated $P$. vivax.

Particular complications of assessment of $P$. vivax trials and follow-up include:

- Genetic diversity of isolates

- Co-infection with $P$. falciparum and the need to treat this infection if it becomes symptomatic

- Assessment of efficacy against relapse infections and the consequent need for long-term follow-up

\section{Conclusions}

WHO has played a major convening role in the past three decades in establishing a normative framework for critical aspects of vaccine development as antigenically-defined subunit vaccines, novel platforms and new adjuvants were introduced for human use, often for the first time in association with malaria vaccine studies. The importance of early and close involvement of scientists, developers, regulators, and public health physicians has enabled continuing close cooperation in later stages as developers evaluate vaccines in ways which will enable technical advisory bodies of WHO to have access to the data that will enable them to provide guidance for country programmes.

This review has focussed on the contributions to clinical trial design. In many cases, the discipline has moved from theory to practice, with large numbers of trials of pre-erythrocytic and asexual stage antigens with different adjuvants now completed and published, so that new protocols can benefit from practical experience added to theoretical considerations. The context has also changed with many trial sites having experienced gratifying reduction in morbidity and mortality, one consequence being the requirements for larger multi-centre studies and complications of interpretation from sites with different intensities of transmission. The reduced transmission has also caused many to focus mainly on the possible contribution of vaccines directed against any stages to reduction of transmission and possible elimination of malaria. Non-falciparum species have taken a backseat role at this stage and ongoing debate continues about the best way of reporting efficacy data. As much is now known and published on pre-erythrocytic and asexual stage vaccine trials, at least to proof of concept stage (probably better described as experimental medicine rather than product development), the review has given greater attention to what lies ahead. The focus is on design of trials for which there is little or no experience, namely trials of vaccines for $P$. vivax, and trials for assessment of reduction of transmission, and for which preferred product characteristics are still the subject of debate (for example with respect to efficacy against hypnozoites or go/no go criteria for transmission blocking studies).

The field is forever changing, with falling transmission leading to changed populations at risk, including older children beyond the age of the EPI schedule or migrants at risk of epidemic malaria. Vaccines that could be used locally for control or elimination of multi-drug resistant malaria require consideration of new product profiles and novel ways of assessing combined efforts with multiple tools for malaria control that call for better ways of measuring effects when transmission is low and nonuniformly distributed.

The review has highlighted the past and ongoing contribution made by WHO in convening groups to address 
key issues for investigators, vaccine developers, regulatory authorities and funders in ensuring the most efficient use of resources for developing much-needed vaccines for use in malaria endemic countries.

\section{Competing interests}

The authors declare that they have no competing interests.

\section{Authors' contributions}

This integration of discussions and recommendations from MALVAC meetings was suggested by VM. The draft was written by GT. All three authors contributed to its revision and approved the final manuscript.

\section{Acknowledgements}

This report contains the collective views of international groups of experts, each group specifically invited by the MALVAC committee for a particular meeting, and does not necessarily represent the decisions, policies or views of the World Health Organization. Graham Brown is the current Chair of the MALVAC committee. We acknowledge the major contribution of Pedro Alonso, the previous MALVAC Chair.

\section{Author details}

${ }^{1}$ Faculty of Infectious and Tropical Diseases, London School of Hygiene \& Tropical Medicine, Keppel Street, London WC1E 7HT UK. ${ }^{2}$ Initiative for Vaccine Research, Department of Immunization, Vaccines and Biologicals, World Health Organization, Avenue Appia 20, 1211-CH 27 Geneva, Switzerland. ${ }^{3}$ Nossal Institute for Global Health, University of Melbourne, Carlton, Victoria 3010 Australia.

Received: 10 May 2013 Accepted: 1 August 2013

Published: 10 October 2013

\section{References}

1. WHO: World Malaria Report 2011. Geneva: World Health Organization; 2011

2. WHO: World Malaria Report 2012. Geneva: World Health Organization; 2012.

3. Rainbow Chart. http://www.who.int/vaccine_research/links/Rainbow/en/.

4. Schwartz L, Brown GV, Genton B, Moorthy VS: A review of malaria vaccine clinical projects based on the WHO rainbow table. Malar J 2012, 11:11.

5. Agnandji ST, Lell B, Fernandes JF, Abossolo BP, Methogo BG, Kabwende AL, Adegnika AA, Mordmuller B, Issifou S, Kremsner PG, Sacarlal J, Aide P, Lanaspa M, Aponte JJ, Machevo S, Acacio S, Bulo H, Sigauque B, Macete E, Alonso P, Abdulla S, Salim N, Minja R, Mpina M, Ahmed S, Ali AM, Mtoro AT, Hamad AS, Mutani P, Tanner M, et al: A phase 3 trial of RTS, S/AS01 malaria vaccine in African infants. N Engl J Med 2012, 367:2284-2295.

6. Hill AV: Vaccines against malaria. Philos Trans R Soc Lond B Biol Sci 2011, 366:2806-2814.

7. Pink JR, Kieny MP: 4th meeting on Novel Adjuvants Currently in/close to Human Clinical Testing World Health Organization - Fondation Merieux, Annecy, France, 23-25, June 2003. Vaccine 2004, 22:2097-2102.

8. Tarantola D, Macklin R, Reed ZH, Kieny MP, Osmanov S, Stobie M, Hankins C: Ethical considerations related to the provision of care and treatment in vaccine trials. Vaccine 2007, 25:4863-4874.

9. Laurens MB, Duncan CJ, Epstein JE, Hill AV, Komisar JL, Lyke KE, Ockenhouse CF, Richie TL, Roestenberg M, Sauerwein RW, Spring MD, Talley AK, Moorthy VS: A consultation on the optimization of controlled human malaria infection by mosquito bite for evaluation of candidate malaria vaccines. Vaccine 2012, 30:5302-5304.

10. NIAID/WHO Workshop on Heterologous Prime-Boost Strategies for HIV, Malaria and Tuberculosis Vaccines. 2012. http://www.malariavaccine.org/malvacroadmap.php.

11. Malaria Vaccine Technology Roadmap. http://www.malariavaccineroadmap.net.

12. Moorthy VS, Newman RD, Duclos P, Okwo-Bele JM, Smith PG: Assessment of the RTS, S/AS01 malaria vaccine. Lancet Infect Dis 2013, 13:280-282.

13. Roestenberg M, Bijker EM, Sim BK, Billingsley PF, James ER, Bastiaens GJ, Teirlinck AC, Scholzen A, Teelen K, Arens T, van der Ven AJ, Gunasekera A, Chakravarty S, Velmurugan S, Hermsen CC, Sauerwein RW, Hoffman SL: Controlled human malaria infections by intradermal injection of cryopreserved Plasmodium falciparum sporozoites. Am J Trop Med Hyg 2013, 88:5-13.
14. Moorthy VS, Diggs C, Ferro S, Good MF, Herrera S, Hill AV, Imoukhuede EB, Kumar S, Loucq C, Marsh K, Ockenhouse CF, Richie TL, Sauerwein RW: Report of a consultation on the optimization of clinical challenge trials for evaluation of candidate blood stage malaria vaccines, 18-19 March 2009, Bethesda, MD, USA. Vaccine 2009, 27:5719-5725.

15. Moorthy VS, Reed Z, Smith PG: MALVAC 2008: Measures of efficacy of malaria vaccines in phase $2 \mathrm{~b}$ and phase 3 trials--scientific, regulatory and public health perspectives. Vaccine 2009, 27:624-628.

16. Pinder M, Moorthy VS, Mendis K, Brown GV: MALVAC 2010: Measures of efficacy of anti-malarial interventions against malaria transmission. Geneva, Switzerland; 2010.

17. Sinden RE, Carter R, Drakeley C, Leroy D: The biology of sexual development of Plasmodium: the design and implementation of transmission-blocking strategies. Malar J 2012, 11:70.

18. Farrance CE, Rhee A, Jones RM, Musiychuk K, Shamloul M, Sharma S, Mett V, Chichester JA, Streatfield SJ, Roeffen W, van de Vegte-Bolmer M, Sauerwein RW, Tsuboi T, Muratova OV, Wu Y, Yusibov V: A plant-produced Pfs 230 vaccine candidate blocks transmission of Plasmodium falciparum. Clin Vaccine Immunol 2011, 18:1351-1357.

19. Farrance CE, Chichester JA, Musiychuk K, Shamloul M, Rhee A, Manceva SD, Jones RM, Mamedov T, Sharma S, Mett V, Streatfield SJ, Roeffen W, van de Vegte-Bolmer M, Sauerwein RW, Wu Y, Muratova O, Miller L, Duffy P, Sinden R, Yusibov V: Antibodies to plant-produced Plasmodium falciparum sexual stage protein Pfs25 exhibit transmission blocking activity. Hum Vaccin 2011, 7(Suppl):191-198.

20. Goodman AL, Blagborough AM, Biswas S, Wu Y, Hill AV, Sinden RE, Draper SJ: A viral vectored prime-boost immunization regime targeting the malaria Pfs 25 antigen induces transmission-blocking activity. PLoS One 2011, 6:e29428.

21. Smith T, Schapira A: Reproduction numbers in malaria and their implications. Trends Parasitol 2012, 28:3-8.

22. Pinder M, Moorthy VS, Akanmori BD, Genton B, Brown GV: MALVAC 2009: progress and challenges in development of whole organism malaria vaccines for endemic countries, 3-4 June 2009, Dakar, Senegal. Vaccine 2010, 28:4695-4702.

23. Clyde DF: Immunization of man against falciparum and vivax malaria by use of attenuated sporozoites. Am J Trop Med Hyg 1975, 24:397-401.

24. Rieckmann KH, Beaudoin RL, Cassells JS, Sell KW: Use of attenuated sporozoites in the immunization of human volunteers against falciparum malaria. Bull World Health Organ 1979, 57(Suppl 1):261-265.

25. Hoffman SL, Goh LM, Luke TC, Schneider I, Le TP, Doolan DL, Sacci J, de la Vega P, Dowler M, Paul C, Gordon DM, Stoute JA, Church LW, Sedegah M, Heppner DG, Ballou WR, Richie TL: Protection of humans against malaria by immunization with radiation-attenuated Plasmodium falciparum sporozoites. J Infect Dis 2002, 185:1155-1164.

26. Hoffman SL, Billingsley PF, James E, Richman A, Loyevsky M, Li T, Chakravarty S, Gunasekera A, Chattopadhyay R, Li M, Stafford R, Ahumada A, Epstein JE, Sedegah M, Reyes S, Richie TL, Lyke KE, Edelman R, Laurens MB, Plowe CV, Sim BK: Development of a metabolically active, non-replicating sporozoite vaccine to prevent Plasmodium falciparum malaria. Hum Vaccin 2010, 6:97-106.

27. Epstein JE, Tewari K, Lyke KE, Sim BK, Billingsley PF, Laurens MB, Gunasekera A, Chakravarty S, James ER, Sedegah M, Richman A, Velmurugan S, Reyes S, Li M, Tucker K, Ahumada A, Ruben AJ, Li T, Stafford R, Eappen AG, Tamminga C, Bennett JW, Ockenhouse CF, Murphy JR, Komisar J, Thomas N, Loyevsky M, Birkett A, Plowe CV, Loucq C, et al: Live attenuated malaria vaccine designed to protect through hepatic CD8(+) T cell immunity. Science 2011, 334:475-480.

28. Annoura T, Ploemen IH, van Schaijk BC, Sajid M, Vos MW, van Gemert GJ, Chevalley-Maurel S, Franke-Fayard BM, Hermsen CC, Gego A, Franetich JF, Mazier D, Hoffman SL, Janse CJ, Sauerwein RW, Khan SM: Assessing the adequacy of attenuation of genetically modified malaria parasite vaccine candidates. Vaccine 2012, 30:2662-2670

29. Roestenberg M, Teirlinck AC, McCall MB, Teelen K, Makamdop KN, Wiersma $J$, Arens T, Beckers P, van Gemert G, van de Vegte-Bolmer M, van der Ven AJ, Luty AJ, Hermsen CC, Sauerwein RW: Long-term protection against malaria after experimental sporozoite inoculation: an open-label follow-up study. Lancet 2011, 377:1770-1776.

30. Good MF: A whole parasite vaccine to control the blood stages of Plasmodium: the case for lateral thinking. Trends Parasitol 2011, 27:335-340

31. Gething PW, Elyazar IR, Moyes CL, Smith DL, Battle KE, Guerra CA, Patil AP, Tatem AJ, Howes RE, Myers MF, George DB, Horby P, Wertheim HF, Price RN, 
Mueller I, Baird JK, Hay SI: A long neglected world malaria map: Plasmodium vivax endemicity in 2010. PLoS Negl Trop Dis 2012, 6:e1814.

32. Bernabeu M, Gomez-Perez GP, Sissoko S, Niambele MB, Haibala AA, Sanz A, Thera MA, Fernandez-Becerra C, Traore K, Alonso PL, Bassat Q, Del Portillo HA, Doumbo O: Plasmodium vivax malaria in Mali: a study from three different regions. Malar J 2012, 11:405.

33. Brown GV, Moorthy VS, Reed Z, Mendis K, Arevalo-Herrera M, Alonso P: Priorities in research and development of vaccines against Plasmodium vivax malaria. Vaccine 2009, 27:7228-7235.

34. Mueller I, Moorthy VS, Brown GV, Smith PG, Alonso P, Genton B: Guidance on the evaluation of Plasmodium vivax vaccines in populations exposed to natural infection. Vaccine 2009, 27:5633-5643.

35. The malERA Consultative Group on Vaccines: A research agenda for malaria eradication: vaccines. PLoS Med 2011, 8:e1000398.

doi:10.1186/1475-2875-12-362

Cite this article as: Targett et al:: Malaria vaccine research and

development: the role of the WHO MALVAC committee. Malaria Journal 2013 12:362.

\section{Submit your next manuscript to BioMed Central and take full advantage of:}

- Convenient online submission

- Thorough peer review

- No space constraints or color figure charges

- Immediate publication on acceptance

- Inclusion in PubMed, CAS, Scopus and Google Scholar

- Research which is freely available for redistribution 